\title{
Atmospheric Turbulence Estimates from a Pulsed Lidar
}

\author{
Matthew J. Pruis ${ }^{1}$ and Donald P. Delisi ${ }^{2}$ \\ NorthWest Research Associates, Redmond, WA, 98052-5164, USA \\ Nash'at N. Ahmad ${ }^{3}$ and Fred H. Proctor ${ }^{4}$ \\ NASA Langley Research Center, Hampton, VA, 23681-2199, USA
}

\begin{abstract}
Estimates of the eddy dissipation rate (EDR) were obtained from measurements made by a coherent pulsed lidar and compared with estimates from mesoscale model simulations and measurements from an in situ sonic anemometer at the Denver International Airport and with EDR estimates from the last observation time of the trailing vortex pair. The estimates of EDR from the lidar were obtained using two different methodologies. The two methodologies show consistent estimates of the vertical profiles. Comparison of EDR derived from the Weather Research and Forecast (WRF) mesoscale model with the in situ lidar estimates show good agreement during the daytime convective boundary layer, but the WRF simulations tend to overestimate EDR during the nighttime. The EDR estimates from a sonic anemometer located at 7.3 meters above ground level are approximately one order of magnitude greater than both the WRF and lidar estimates - which are from greater heights - during the daytime convective boundary layer and substantially greater during the nighttime stable boundary layer. The consistency of the EDR estimates from different methods suggests a reasonable ability to predict the temporal evolution of a spatially averaged vertical profile of EDR in an airport terminal area using a mesoscale model during the daytime convective boundary layer. In the stable nighttime boundary layer, there may be added value to EDR estimates provided by in situ lidar measurements.
\end{abstract}

\section{Nomenclature}

$\begin{array}{ll}\alpha & =\text { Kolmogorov constant } \\ \varepsilon & =\text { eddy dissipation rate (same as EDR) } \\ \text { ASOS } & =\text { Automated Surface Observing System } \\ \text { CTI } & =\text { Coherent Technologies, Inc. } \\ \text { EDR } & =\text { eddy dissipation rate (same as } \varepsilon \text { ) } \\ k & =\text { wavenumber } \\ \text { lidar } & =\text { LIght Detection And Ranging } \\ \text { LOS } & =\text { Line of sight } \\ \text { NCAR } & =\text { National Center for Atmospheric Research } \\ \text { TKE } & =\text { turbulence kinetic energy } \\ S & =\text { turbulent energy spectrum } \\ \text { WRF } & =\text { Weather Research and Forecast model }\end{array}$

\section{Introduction}

$\mathrm{O}$ $\mathrm{NE}$ of the important factors affecting the time evolution of the strength and transport of aircraft wake vortices is the strength of the ambient atmospheric turbulence. Unfortunately, it is difficult to obtain in situ observations of the ambient turbulence at the heights of the aircraft in the airport terminal area. Measurements of the eddy dissipation rate (EDR) are often obtained using high frequency wind data from anemometers located less

\footnotetext{
${ }^{1}$ Research Scientist, NWRA, $4118148^{\text {th }}$ Ave NE, Redmond, WA 98052. Member, AIAA.

${ }^{2}$ Senior Research Scientist, NWRA, $4118148^{\text {th }}$ Ave NE, Redmond, WA 98052. Senior Member, AIAA.

${ }^{3}$ Research Aerospace Engineer, NASA, Hampton, VA 23681. Senior Member, AIAA.

${ }^{4}$ Senior Research Scientist, NASA, Hampton, VA 23681. Senior Member, AIAA.
} 
than 30 meters above ground level (e.g., Campbell et al. ${ }^{1}$ ). To extend these observations to the heights of the aircraft requires the use of similarity theory ${ }^{2}$.

In this study, we estimate EDR at the heights and locations of aircraft vortex wake measurements using line of sight (LOS) winds from a scanning coherent pulsed lidar. EDR estimates from a pulsed lidar have the advantage of being local and have spatial and temporal resolution that may be able to resolve scales that are appropriate for aircraft wake vortex studies. We examine two different methods to estimate EDR from the lidar. These two methods use either temporal data (to estimate temporal spectra) or a combination of spatial and temporal data (to estimate structure functions). We compare the EDR obtained from both methods.

We also compare the EDR estimates from the lidar with the EDR estimates from a mesoscale model simulation. The mesoscale model used in this analysis was the National Center for Atmospheric Research's (NCAR) Weather Research and Forecast (WRF) model. The EDR is diagnosed from the WRF model output of turbulence kinetic energy (TKE) and the master length scale. The advantage of the mesoscale model estimates is that they can provide better spatial coverage of the terminal area than the lidar measurements, which are taken in a single measurement plane, as well as forecasts for planning operations.

We have found that the estimates of EDR from the WRF model simulations agree well during the daytime convection regime. This time period tends to correspond with the peak in number of aircraft that are approaching, or leaving the terminal area. Differences in the EDR estimates are much larger during the stable nocturnal boundary layer. Turbulence in the very-stable nocturnal boundary layer is weak and the WRF model estimates of TKE typically reach default thresholds during this time interval and lead to a biased estimate of EDR.

Finally, we compare how well the EDR estimates from both the lidar and the WRF model compare to estimates of EDR from other methods. The two additional methods we examine are the EDR estimates obtained from a sonic anemometer located on a 7.3 meter tall staff and the EDR estimates obtained by assuming the time of the last vortex observation with the lidar corresponds to the vortex linking time.

These comparisons show that the EDR estimates from the sonic anemometer are approximately one order of magnitude greater than both the WRF and lidar estimates during the daytime convective boundary layer and substantially greater during the nighttime stable boundary layer. These differences are not unexpected since the sonic anemometer was closer to the ground, where the turbulence is expected to be stronger. The estimates of EDR obtained from the aircraft wake vortex observations tend to approximately one order of magnitude lower than all the other methods examined. There is a large amount of scatter in the EDR estimates obtained from the aircraft observation time, but it is unclear why there exists a consistent bias in this data set.

Estimates of EDR from the temporal spectra of the lidar velocity estimates show fine-scale layering of the turbulence structure and intermittently isolated regions of enhanced turbulence. These small scale structures cannot be observed with either the WRF model, which is of much larger scales, or from the single point sonic anemometer. The ability to study the small-scale spatial structure of the turbulence may be important in understanding observed wake behavior.

\section{Data Set}

A comprehensive field experiment to measure wake vortices and the ambient meteorological conditions was conducted at the Denver International Airport from August 26 to September 20, 2003. The experiment was sponsored under the NASA Wake Vortex Avoidance Project. In this project, several sensor systems were deployed to collect meteorological data to characterize the effect of atmospheric conditions on the behavior of wake vortices and the ability to observe them. The meteorological measurements were conducted at a location under the flight path of runway 16L, approximately two nautical miles from the runway threshold. At this location arriving aircraft have a nominal altitude of approximately 212 meters. Meteorological sensors included (1) a meteorological tower equipped with R.M. Young Gill propeller anemometers at three heights and a Vaisala temperature and relative humidity sensor, (2) an AeroVironment Sodar, (3) a Metek ultrasonic anemometer, (4) a Kipp \& Zonen MTP5 temperature profiler, and (5) two lidars, a CTI WindTracer® pulsed Doppler lidar as well as the MIT Lincoln Laboratory continuous wave lidar. In this study we use data from the CTI lidar and the Metek ultrasonic anemometer shown in Figure 1. To illustrate the results, we will focus on September 19. We will then show results covering the period of the wake experiment when there were both sonic anemometer and pulsed lidar data available.

\section{Methods}

In this study, five methods for estimating EDR are examined. Each of these methods has been previously described in literature so we only briefly review the methods below and describe some of the limitations of each of the methods. 

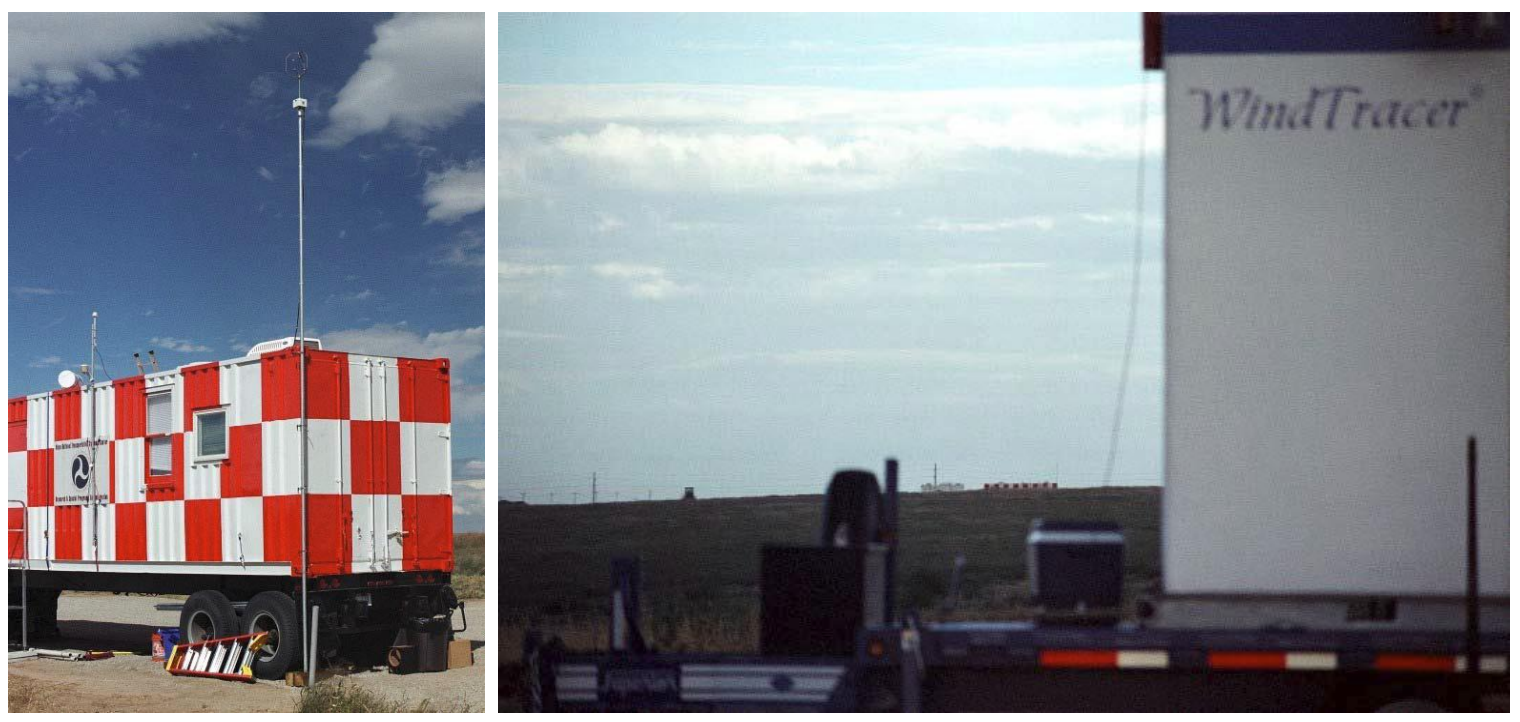

Figure 1. (a) A Metek ultrasonic anemometer was mounted on the NASA-DOT Volpe Instrumentation trailer at a height of 7.3 above ground level and approximately 3.2 meters above the top of the trailer. (b) The CTI WindTracer ${ }^{\circledR}$ pulsed Doppler lidar was located approximately 1100 meters west of the Metek ultrasonic anemometer.

\section{A. Sonic Anemometer at $7.3 \mathrm{~m}$}

A Metek ultrasonic anemometer was deployed at 7.3 meters height above ground mounted on the NASA-DOT Volpe Instrumentation Trailer. The sensor was approximately 3.2 meters above the top of the trailer (Figure 1). The ultrasonic anemometer sampled data at $10 \mathrm{~Hz}$.

The standard method for estimating the rate of dissipation kinetic energy from high-frequency measurements of 3-dimensional velocities typically involves dividing the data into small overlapping windows from which an EDR estimate is derived. Following the common practice, co-ordinate transformation is applied to the three components of the wind to align the longitudinal axis with the mean wind direction, and there is a transformation of the velocity spectra into the wavenumber domain.

It is assumed that the production of turbulent kinetic energy is dominated by large eddies, which then decay into smaller and smaller eddies (the inertial sub-range) until the length scales are small enough for the kinetic energy to dissipate into heat by molecular diffusion in the viscous sub-range (scales on the order of centimeters or less). If the turbulence is homogeneous and isotropic then the Kolmogorov hypothesis states that within the inertial subrange, the statistical representation of the turbulent energy spectrum $S(k)$ is given by:

$$
S(k)=\alpha \varepsilon^{2 / 3} k^{-5 / 3}
$$

where $\alpha=0.55$ is the Kolmogorov constant for longitudinal wind spectra ${ }^{3}$ and $=0.55 \times 4 / 3$ for the transverse and vertical wind spectra, $\varepsilon$ is the eddy dissipation rate and $k$ is the wavenumber, which can be related to a length scale, $L(k=2 \pi / L)$ by invoking Taylor's hypothesis of frozen turbulence ${ }^{4}$. A $-5 / 3$ power-law can be fit to the portion of the observed spectrum that lies within the inertial sub-range to estimate $\varepsilon$ from eqn. (1).

For the sonic anemometer data, the data were broken into 30-minute windows. For each window, a power spectrum was computed and an EDR value was estimated using an iterative RMSE minimization scheme, where the fit is required to span one-third of the observed wavenumber space.

This method of estimating the EDR is most suitable for convective well-mixed boundary layers, where the scales of motion remain well within the inertial sub-range. Caution must be applied when estimating dissipation rates in more quiescent conditions when surface winds may be variable ${ }^{5}$. Estimates of EDR during the nighttime stable boundary layer may become even more uncertain, since eddies generated near the surface are constrained by thermal stratification $^{6}$ and depart from isotropy.

Several quality control parameters were employed to remove time periods of unusual behavior from the time series. The principal goal of the quality control parameters is to identify, and subsequently allow for the removal of, 
sections of data where instrument problems or unusual atmospheric conditions indicate that accurate estimates of the turbulent eddy dissipation rate is not possible ${ }^{5}$. For example, data spikes which can be caused by random electronic spikes in the data monitoring and recording systems were removed using a 3-point running median filter. Higher moment statistical flags based on the skewness and kurtosis of the data samples were set to detect possible instrument or recording problems and physical but unusual behavior. Discontinuities in the data were identified using the Haar transform which calculates the difference in some quantity over two half-window means ${ }^{5}$. The goal of the Haar transform test is to detect sections of the data where discontinuities lead to semi-permanent changes as opposed to sharp changes associated with smaller-scale fluctuations. Finally, the data was examined for periods of nonstationarity. Nonstationary records most often occur when there is a weak large-scale flow and yet significant smaller scale fluctuations. In these cases, the length scale estimated for the frozen turbulence assumption, which is based on the speed of the vector averaged wind, will be different from that computed with the average wind speed.

Figure 2 shows four samples of these spectral fits taken throughout the day and includes examples in both the daytime convective boundary layer and the evening stable boundary layer. It is easy to see the daytime convective period in the longitudinal winds which show large oscillations with 2-8 minute periods during the convective periods. High frequency variability in the winds in the nighttime samples also can be intense, but the largeamplitude, lower-frequency oscillations tend to be suppressed in the nighttime stable boundary layer. An example of a time period where the data was flagged for potentially being nonstationary can be seen in the third spectra shown at the time of 15:15 UTC. The EDR estimate for this time period was not included in the study since it was quality control flagged for the crosswind relative nonstationarity. As can be seen in the figure, the mean longitudinal winds used for estimating the wavenumber are low during the first half of this record period and may lead to inaccurate turbulence estimates (even though the spectra shows a good $-5 / 3$ slope region). We found that time periods when the mean longitudinal wind is less than $2 \mathrm{~m} / \mathrm{s}$ are frequently flagged for either alongwind, crosswind, or vector wind relative nonstationarity.

\section{B. Lidar Structure Functions}

The usage of spatial structure functions derived from pulsed Doppler lidar measurements has been extensively documented in the literature ${ }^{7-12}$ and has shown to be a promising method for estimating EDR at an accuracy that is desired in wake vortex studies ${ }^{11,12}$. Recently, Pruis and Delisi ${ }^{12}$ applied this technique to estimate vertical profiles of EDR from a NASA wake vortex measurement campaign in 2006 at Denver International Airport. They compared the results with EDR profiles obtained using two sonic anemometers on a tower and extrapolated to the heights of the observed aircraft wakes using similarity theory ${ }^{1,2}$. They found good agreement between these two methods of estimating the EDR vertical profiles. Likewise, a recent experimental result of Banakh, et al. ${ }^{11}$ shows the lidar EDR estimates obtained from spatial structure functions had small bias and relative errors not greater than $25 \%$ when compared with sonic anemometers mounted on a 120 meter vertical tower in a daytime convective boundary layer.

The methodology described in Pruis and Delisi ${ }^{12}$ was applied to this data set. One drawback of this method is the assumption that the turbulence is isotropic and stationary in both time and space over reasonably long time and space intervals. In reality, turbulence can be highly localized in both time and space. For example, the stable nocturnal boundary layer is often characterized by abrupt changes in variance, as much as an order of magnitude, and frequently patches of enhanced turbulence may be isolated into thin layers or pockets ${ }^{13,14}$.

Figure 3 shows EDR estimated from the structure function on 19 September 2003. These estimates show much larger variability throughout the day than the EDR estimated from the sonic anemometer. As expected, these estimates show much lower EDR during the nighttime stable boundary layer than during daytime convective boundary layer. There is an indication of an "upside down" turbulence profile around 0600 UTC. This type of profile is typically caused by mechanical production of turbulence due to shear aloft, which then propagates downwards toward the surface.

\section{Lidar Temporal Spectra}

Using the spectra computed from the LOS velocity estimates from a vertically-pointing Doppler lidar was described by Banakh, et al. ${ }^{11}$ and more recently O'Connor, et al. ${ }^{7}$ We have modified their techniques in that we are using a pulsed Doppler lidar in a scanning mode and therefore obtain a two-dimensional map of the EDR instead of a single vertical profile. The scanning mode also provides a high-quality estimate of the time-varying horizontal inplane wind and does not require a separate measurement of the mean wind speed. To fit the spectra, we followed the same methodology used for the sonic anemometer data described above.

One shortcoming of this method is that the revisit time is infrequent. For this experiment, the revisit time was six seconds. Thus, this method is only suitable for retrieval of dissipation rates when the observable relevant scales of motion remain within the inertial sub-range.

4

American Institute of Aeronautics and Astronautics 

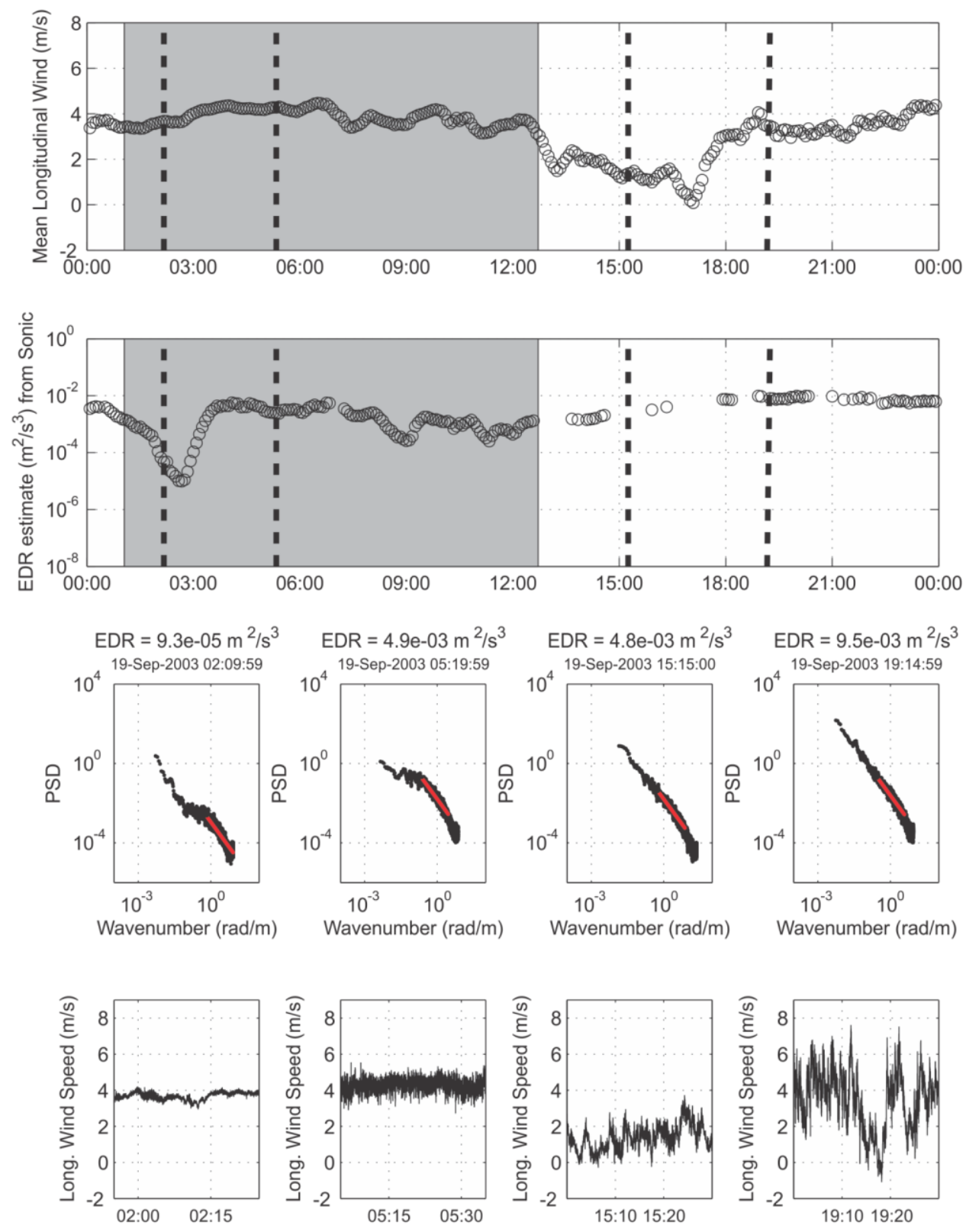

Figure 2. Mean longitudinal wind speed (top panel) and EDR estimated from the $10 \mathrm{~Hz}$ sonic anemometer located at 7.3 meters height above the ground (second panel) on 19 September 2003. The EDR estimate shown is the geometric mean of the estimates obtained from the longitudinal, transverse and vertical wind spectrum. The shaded region represents the local nighttime hours. Samples of the spectra computed using 30 minutes of data at four different times in the day are shown in the middle four panels. The black vertical dashed lines in the top two panels indicate the times of the spectra. The red lines are $-5 / 3$ powerlaw fits to the spectra. The bottom four panels show the longitudinal wind corresponding to the spectra. Times shown are UTC. Local time is UTC -6 hours.

Figure 4 shows the EDR estimates using the temporal spectra method with the lidar data for the day of 19 September 2003. The profiles and the heat map of the variation of EDR over the day is very similar to those estimated using the spatial structure functions. There is a tendency for the estimates of the EDR from the temporal spectra to be higher than that estimated from the spatial structure functions. This may in part be because of the increased sensitivity of the temporal spectra methodology to local turbulence anomalies. 

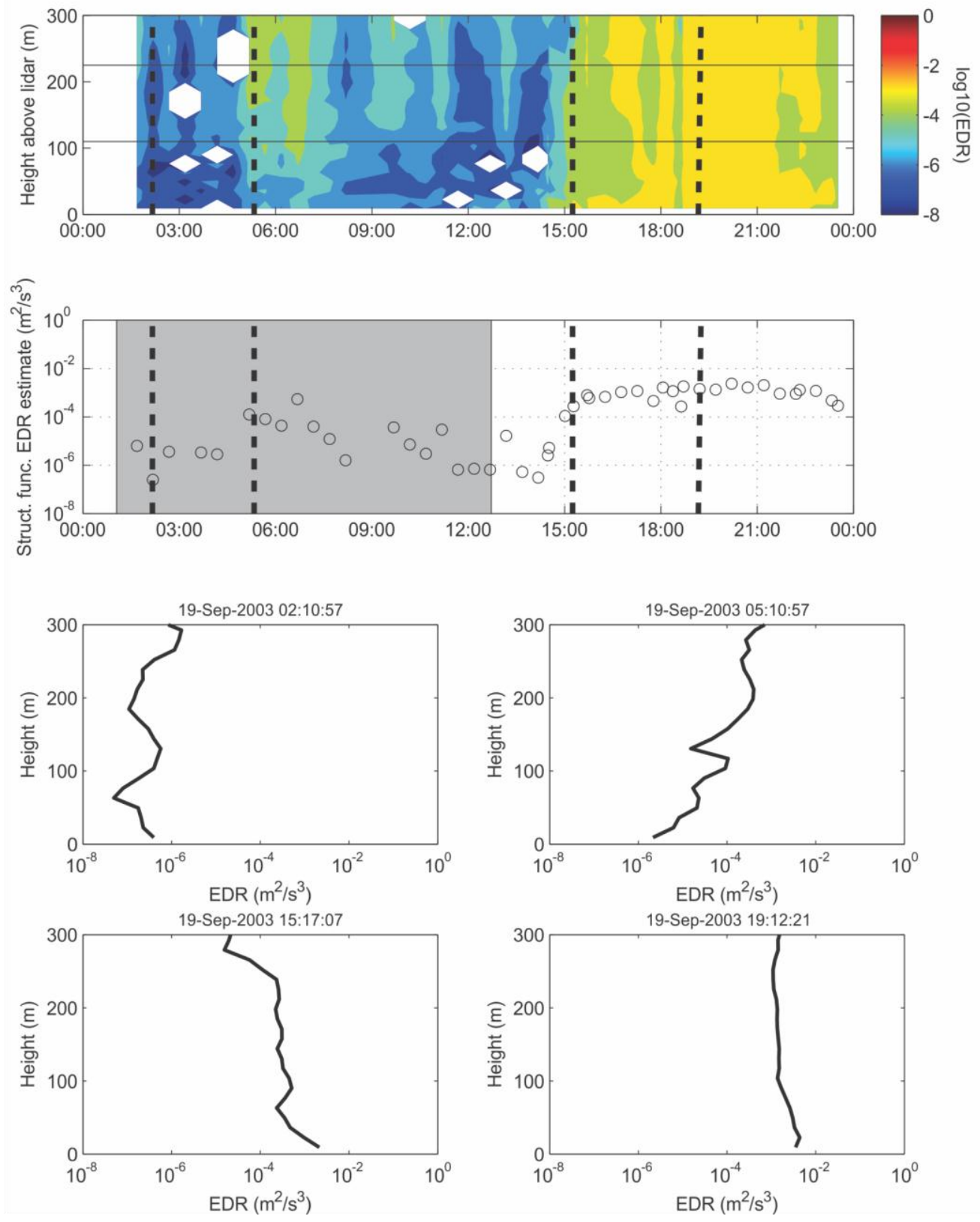

Figure 3. EDR estimated from lidar data using spatial structure functions every 30 minutes on 19 September 2003. The heat map in the upper panel shows the temporal variability in the EDR estimates. The second panel shows the geometric mean of the lidar structure function EDR estimates over the heights of 110 to 225 meters. The shaded region represents the local nighttime hours. Examples of the individual EDR profiles at four different times of the day are shown in the bottom four panels. These times are marked on the upper two panel with dashed vertical lines. Times shown are UTC. Local time is UTC - 6 hours.

To create a single vertical profile out of a two-dimensional map of turbulence, the data were binned every 13.5 meters in the vertical and averaged. Much like in Figure 3, the heat map plot in Figure 4 shows a strong variability in the EDR estimates throughout the day with much lower values in the evening stable boundary layer than in the daytime convective boundary layer.

Figure 4 also shows an "upside down" turbulence profile around 0600 UTC, where it appears that turbulence generated aloft propagates down towards the surface. Overall, the temporal spectra and the structure function 

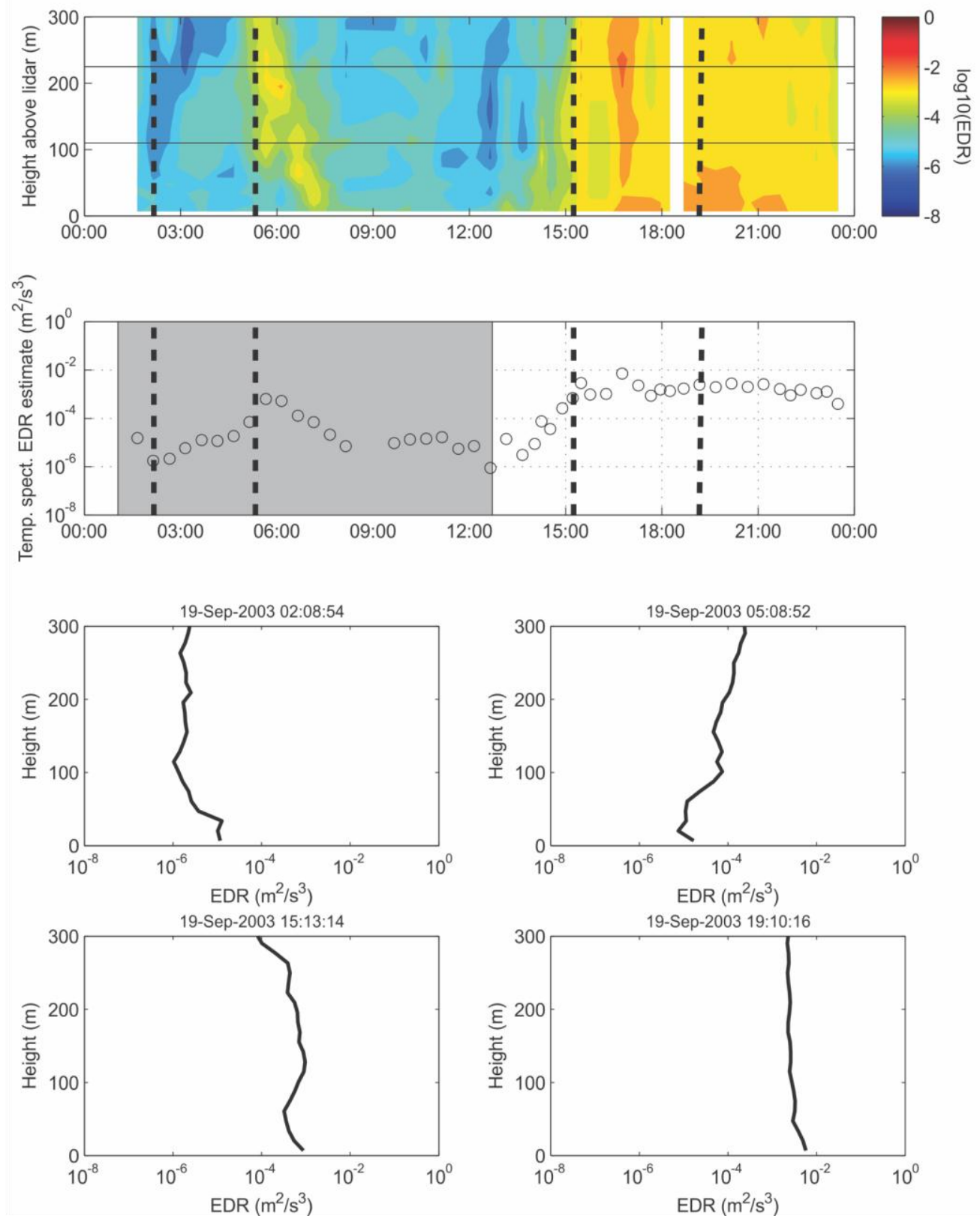

Figure 4. The EDR estimated from lidar data using the temporal spectra every 30 minutes. The data was binned every 13.5 meters in the vertical and averaged to create the vertical profiles seen in the heat map in the upper panel. The second panel shows the time series of the geometric average of the profiles over the height interval of 110 to 225 meters. The shaded region represents the local nighttime hours. The lower four panels show individual profiles extracted at the times shown in the upper panels by the vertical dashed lines. Local time is UTC - 6 hours.

estimates show similar trends, but the temporal spectra estimates of EDR tend to be slightly higher than the estimates from the spatial structure functions.

Figure 5 shows the additional spatial information that is available with the temporal spectra estimates. Shown are the two-dimensional heat maps of turbulence at the four times of the profiles shown in Figure 4. These times are approximately the same times as the individual profiles shown in Figure 3. The first two maps correspond to times when the boundary layer is stable and show fine scale layering of the turbulence with height from the ground to $\sim 60$ meters altitude. Successive maps show these layers frequently maintain their integrity over several observation 

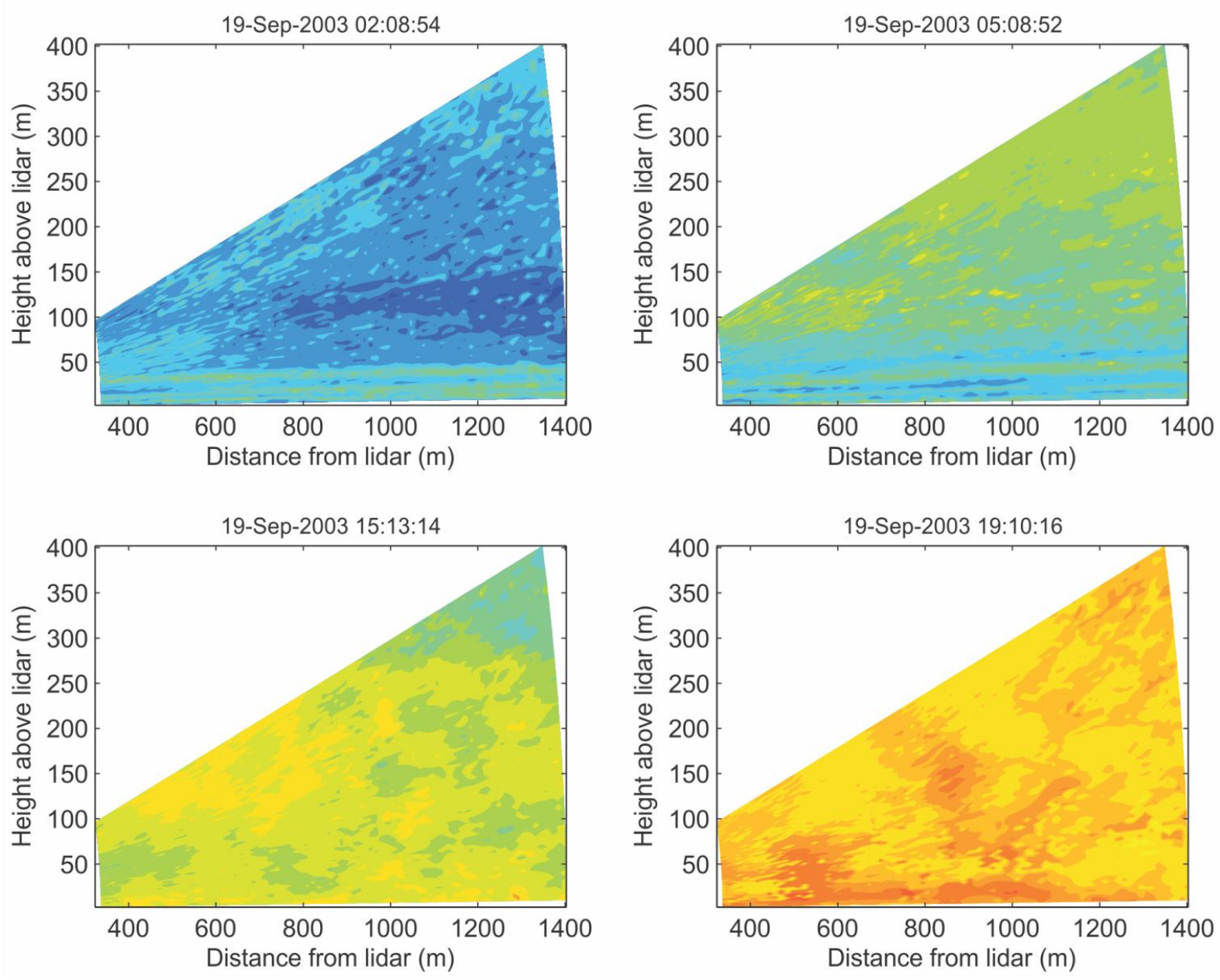

Figure 5. Example two-dimensional heat maps of the EDR estimates from lidar data using the temporal spectra. The EDR maps correspond to the four vertical profiles that are shown in Figure 4. The colormap is the same as used in Figures 3 and 4. The upper two panels show two times during the nighttime stable boundary layer. The lower two panels are two examples from the daytime convective boundary layer. Local time is UTC - 6 hours.

intervals (thus they are sometimes persistent on the timescale of hours) and may move up or down over time. It is also sometimes observed that the layers thicken and become more diffused throughout the night. The difference of the turbulence within these layers can be as much as ten times the turbulence estimated outside of these layers.

\section{Mesoscale Modeling}

Ahmad and Proctor ${ }^{15}$ have previously described the use of WRF model outputs of TKE and the master length scale which are used to compute the EDR in a post-processing step. A similar methodology was used here for application to the Denver Airport. The computation domain was comprised of two nests. The outermost nest had a spatial resolution of $12 \mathrm{~km}$ while the innermost nest had a resolution of $4 \mathrm{~km}$. There were 60 vertical levels in the simulation with the finest resolution near the ground. High-resolution terrain and landuse datasets were included in the simulation. The simulations used the Rapid Radiative Transfer Model (RRTM) longwave and the Goddard shortwave schemes for radiative transfer. The planetary boundary layer scheme was the Mellor-Yamada-Janjic (MYJ) where the thresholds were modified. The simulations used a $5^{\text {th }}$-order upwind scheme in the horizontal and a $3^{\text {rd }}$-order upwind scheme in the vertical. The model was initialized at 0000 UTC 19 September 2003 using data from the National Climate Data Center's North American Regional Reanalysis.

Figure 6 shows the WRF estimates of EDR on 19 September 2003. The trends throughout the day mirror the trends seen in the EDR estimates from lidar, but tend to be biased high during the nighttime stable boundary layer. 

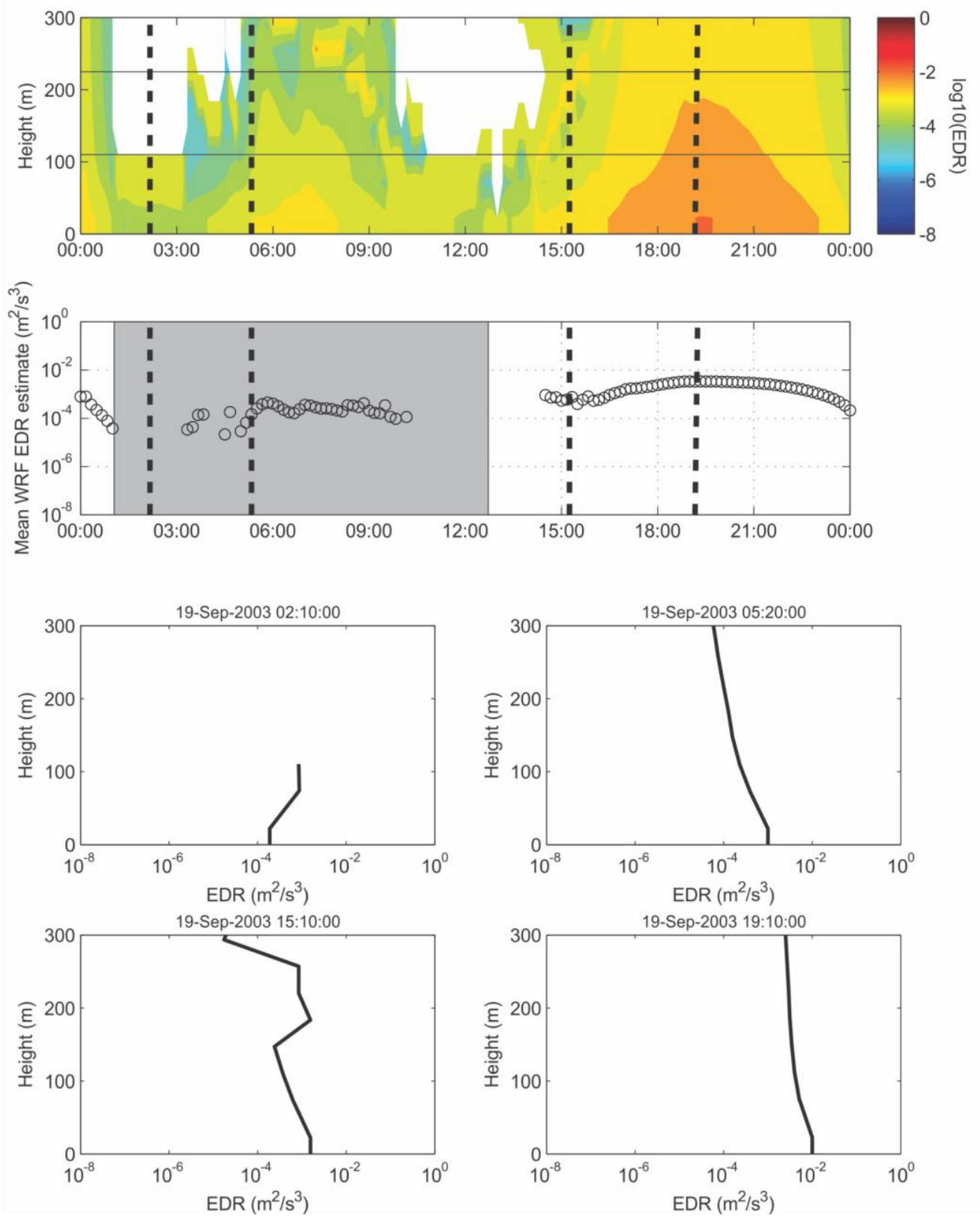

Figure 6. Estimates of EDR generated from the WRF mesoscale model for 19 September 2003 at Denver International Airport. The heat map in the upper panel shows the temporal variability in the EDR estimates. The second panel shows the geometric average of the profiles over the height interval of $110 \mathrm{~m}$ to $225 \mathrm{~m}$. The blank sections of the heat map and the times where there are no estimates of the EDR in the second panel correspond to times and locations where the minimum TKE threshold was reached. Examples of the individual vertical profiles of the EDR from four different times of the day are shown in the bottom four panels. Local time is UTC -6 hours.

During the daytime convective boundary layer the estimates are similar to the estimates from the lidar but, as expected, show much smoother variations in both height and time than is observed with the in situ lidar data.

Overall, we find that the WRF model is able to capture the main features of the complete diurnal cycle such as the development of the nighttime stable boundary layer and the transition of the nighttime stable boundary layer into the daytime convective boundary layer. The forecasts of surface temperature and winds compared favorably both qualitatively and quantitatively with observations from ASOS. The EDR predictions compare well with the other 


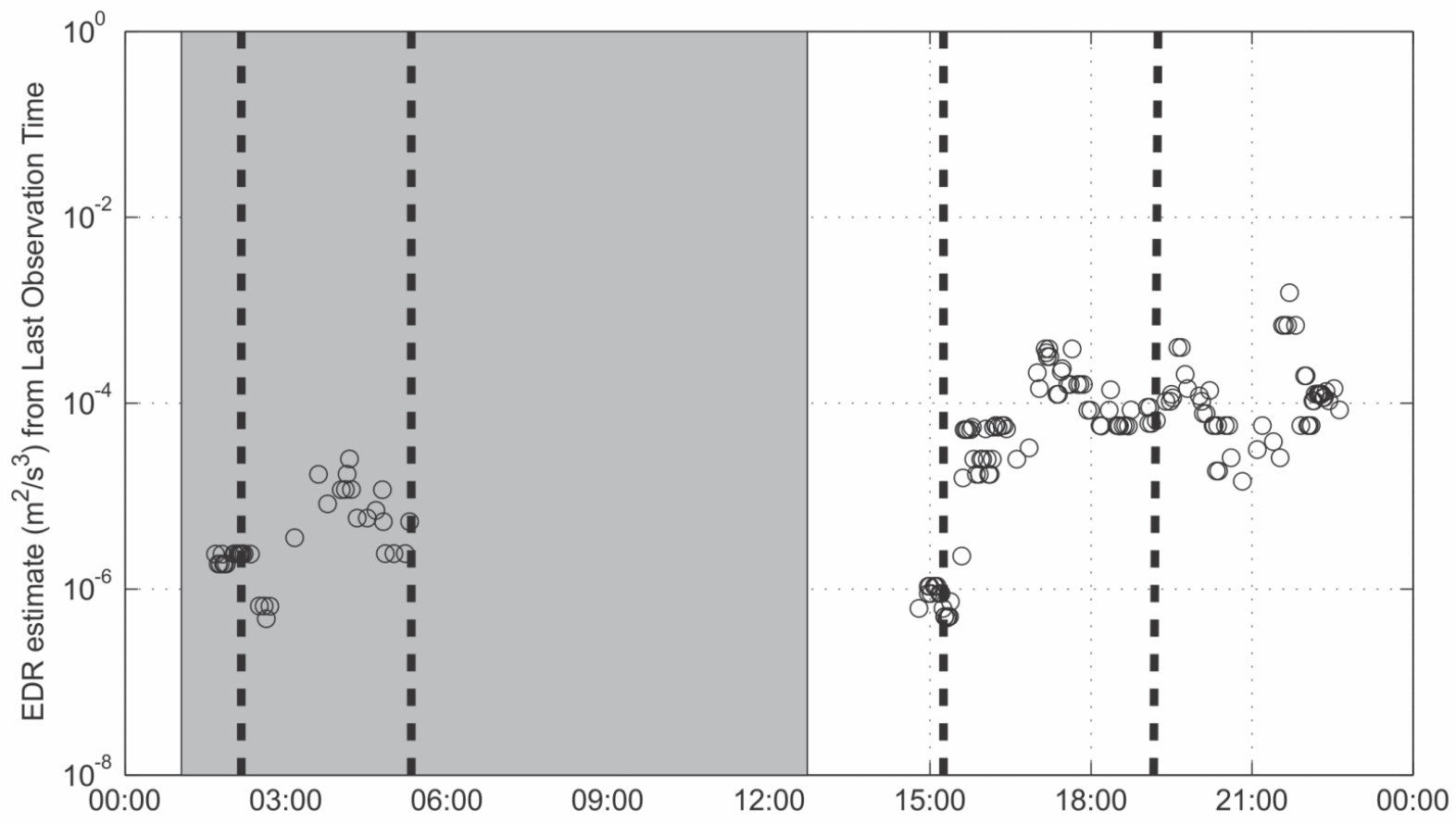

Figure 7. EDR estimates from the time of last observation on 19 September 2003 at Denver International Airport. The time of last observation was assumed to be the linking time and Sarpkaya's relationship ${ }^{16}$ between linking time and the atmospheric EDR was used to generate these EDR estimates. Thus times correspond to an aircraft landing. The EDR estimates plotted is the 30-minute geometric median EDR since Pruis and Delisi ${ }^{12}$ previously showed that the Sarpkaya time to linking corresponds to the time when $50 \%$ of the wakes are no longer observed. The shaded region represents the local nighttime hours. The black vertical dashed lines are the same times shown in Figures 2-6 and are shown for reference only. Times shown are UTC. Local time is UTC - 6 hours.

estimates of EDR during the daytime convective boundary layer, but large biases are observed during the nighttime stable boundary layer. The MYJ PBL scheme tends to overestimate EDR during the nighttime stable boundary layer. To further evaluate the performance of the scheme will require simulations covering a larger time interval with a more diverse set of meteorological conditions and may require additional tuning of the model and algorithms.

\section{E. Observations of Wake Vortex Lifetimes}

As a final method to estimate the atmospheric EDR, we use the length of time that the wakes were observed with the lidar. Sarpkaya ${ }^{16}$ proposed a functional relationship whereby the linking time of vortices could be estimated directly from the ambient EDR. This relationship has been supported by additional aircraft measurements, numerical studies ${ }^{18}$ and laboratory experiments ${ }^{19}$. Recently, Pruis and Delisi ${ }^{17}$ re-examined Sarpkaya's functional relationship using 2006 lidar data at Denver International Airport and found that, after accounting for instrumentation and experimental design factors, the Sarpkaya functional relationship closely tracked the time when $50 \%$ of vortex tracks were no longer observed.

One uncertainty associated with this methodology is the assumption that the observation lifetime corresponds to linking time. However, it is reasonable that wake vortices may be observed for a time interval that is both less than or greater than the linking time. Importantly, it has not been shown that a lidar cannot observe vortices after linking for as long as the vortex ring remains in the line of sight of the lidar.

Figure 7 shows the estimates of EDR obtained using Sarpkaya's relationship between linking time and the atmospheric turbulent eddy dissipation rate and the assumption that the time of the last vortex observation with the lidar coincides with the time of vortex linking. Since the analysis of Pruis and Delisi showed that the Sarpkaya relationship corresponded to the time when $50 \%$ of the vortex tracks were no longer observed, the data was filtered with a 30-minute running geometric median. Actual scatter in the individual EDR estimates from this method tends to show substantial scatter. 

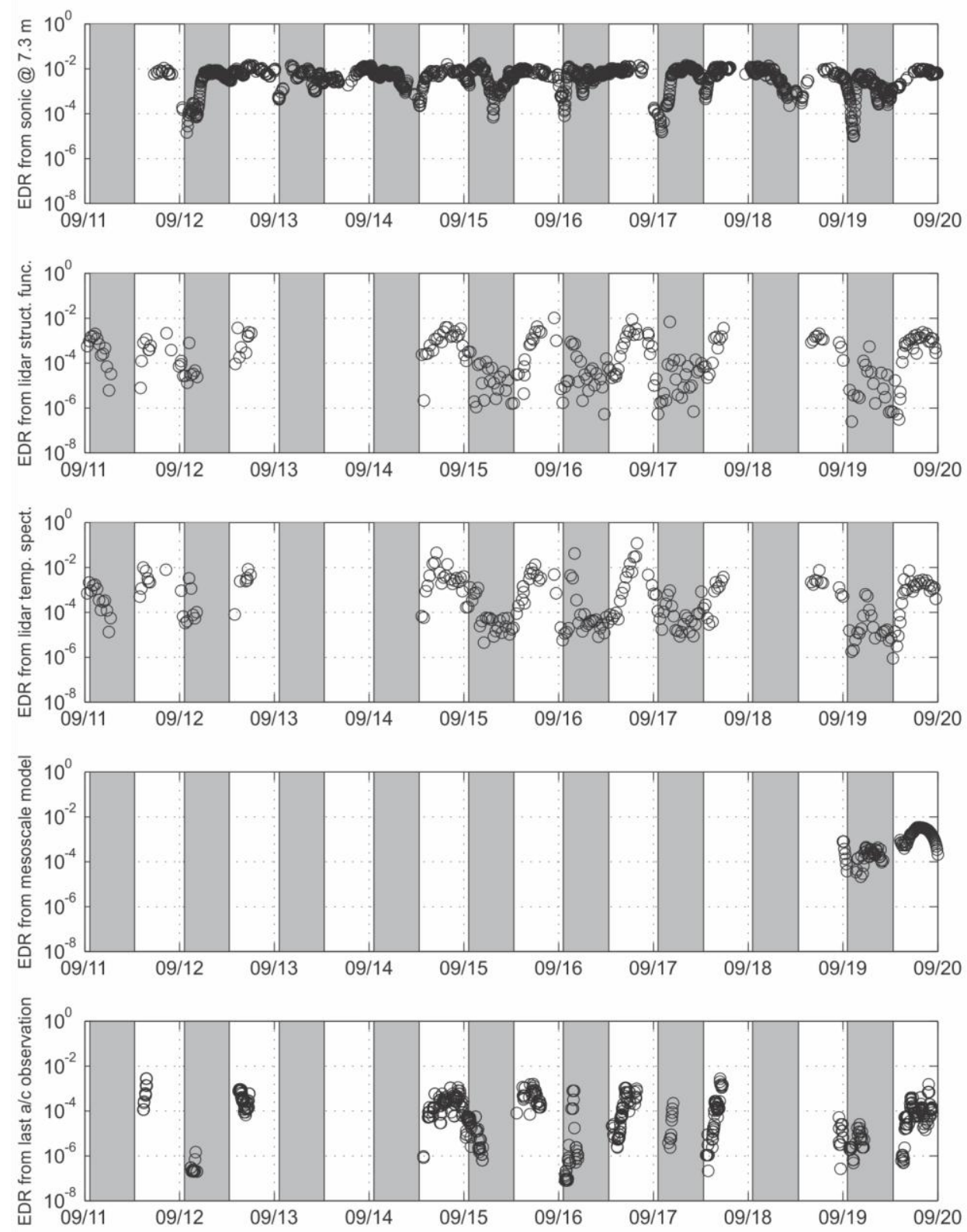

Figure 8. Comparison of EDR estimates over a nine-day period during the experiment. The shaded regions indicate the local nighttime hours. Sonic anemometer measurements at 7.3 meters height above ground level (top panel) was usually available during this interval and generally has the highest EDR estimate, which is expected since the measurement is closer to the ground than the other estimates. The estimates shown for the lidar and WRF (second, third, and fourth panels) are the geometric average over the heights of 110 to 225 meters. The large range in EDR indicates how much EDR is estimated to vary with height at different times of the day. The EDR estimates from Sarpkaya's relationship (bottom panel) generally track the lidar and WRF estimates, but are typically lower than the other EDR estimates.

EDR estimates from this methodology were only used when the lidar was able to track a trailing vortex pair and that vortex pair stayed within the lidar observation region until it was no longer tracked. As such these estimates tend to correspond to periods when the local winds are low. Estimates of EDR from this methodology appear to be approximately one order of magnitude lower than the estimates obtained using the lidar directly. Similar to the other 


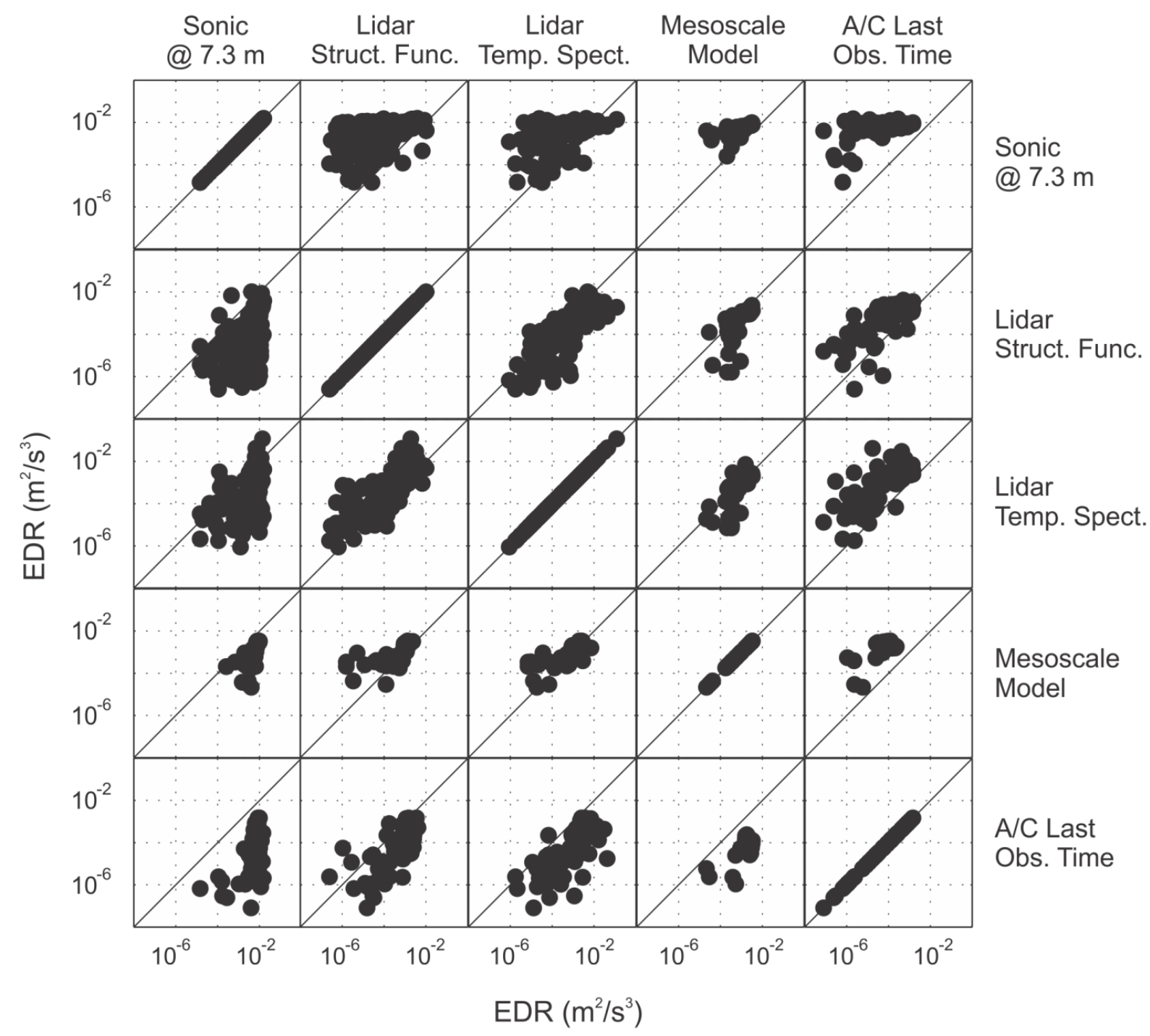

Figure 9. Comparison of EDR estimates over a nine-day period during the experiment. Each method is plotted against itself and all the other EDR estimation methods described in this paper. The estimates shown for the lidar and WRF are the geometric average over the heights of 110 to 225 meters, which is the typical height range where the trailing aircraft vortices were observed. The estimates from the sonic anemometer located at $\mathbf{7 . 3}$ meters do not correlate well with any of the other methods, except that the estimates of all the methods are high in the daytime convective boundary layer. The lidar structure function method tends to produce estimates that are 0.5 to 1 decade lower than the estimates from the estimates from the lidar temporal spectra method. The WRF mesoscale model estimates correlate well with the lidar and aircraft observation time estimates, except that WRF does not predict the very low EDR values in the nighttime stable boundary layer. The estimates from the last observation time of the trailing vortex pair produce lower EDR estimates than the other methods.

EDR estimation methods, the estimates show higher ambient turbulence during the daytime and lower estimates during the nighttime stable boundary layer.

\section{Discussion}

Figure 8 shows a scatter plot of the results of all of the methods discussed over a nine day period when the sonic anemometer was available. All of the methods show the same trends in EDR with the exception of the sonic 
anemometer measurements which, at 7.3 meters, are much higher than the other estimates during the nighttime stable boundary layer and tend to stay higher for 2 to 3 hours after sunrise.

To more closely examine how these methods compare to one another, Figure 9 shows cross plots of all the EDR methods. EDR estimates for each method were interpolated, using nearest-neighbor, onto the 30-minute time grid. The EDR estimates from the lidars and WRF were averaged (geometric) over the height range of 110 to 225 meters because that is the nominal height range traversed by the trailing aircraft vortex pairs in this dataset.

The cross plots in Figure 9 show that none of the methods exhibit much correlation with the EDR estimates obtained from 7.3 meter sonic anemometer, except that during the daytime convective boundary layer when all the methods predict high atmospheric eddy dissipation rates. This result is expected since the sonic anemometer is much closer to the ground than the other estimates.

The lidar structure function method tends to produce EDR estimates that are $1 / 2$ to 1 decade less than the estimates from the lidar temporal spectra method. The lidar structure function estimates tend to be of similar magnitude greater than the estimates from the aircraft last observation time.

The WRF mesoscale model estimates correlate well with the estimates from the lidar and the last observation time of the wake for higher turbulence levels, but the estimates from WRF are biased high during the stable nocturnal boundary layer. Additional study is needed over a longer time interval and covering a more diverse set of atmospheric conditions to better quantify the biases and accuracy of the mesoscale model at estimating EDR at the altitudes and time scales that are relevant to aircraft wake studies in the airport terminal area.

The EDR estimates from the last vortex observation are substantially lower than the lidar estimates, even during the daytime convective boundary layer. This may indicate that vortices are being tracked after they have linked or it may indicate a sampling bias where very short lived vortex tracks associated with higher atmospheric turbulence levels are not tracked well by the vortex processing algorithm used in this study. In any case, this result warrants additional study.

\section{Conclusions}

The eddy dissipation rate is an important parameter in describing the behavior of wake vortices. The ability to measure and predict EDR along the flight path in the vicinity of airports improves our ability to predict the location and strength of the aircraft's trailing wake vortices. In this study, five different methods were used to estimate the eddy dissipation rate in the airport environment. All of these methods provide consistent estimates of EDR during the daytime convective boundary layer. The sonic anemometer gives higher estimates than the other methods, but this is expected due to its close proximity to the ground.

The temporal spectra and the spatial structure function methods of estimating EDR from lidar provide similar estimates. The structure function methodology tends to provide lower EDR estimates and exhibits greater variability. A benefit of the temporal spectra is the ability to visualize fine-layering and regions of enhanced turbulence. The EDR estimates from the WRF mesoscale model tend to be biased high compared to the lidar estimates. The bias is particularly strong during the nighttime, stable boundary layer. Estimates of EDR derived from using the maximum observation time of the vortices and Sarpkaya's function relationship relating the time to linking to the intensity of the atmospheric turbulence are similar to the lidar EDR estimates, but predict much lower turbulence levels. This might correspond to the tracking of vortices after the time of linking or might represent a sampling bias where vortices are not tracked well when the turbulence levels are high and justifies further study. With additional research and analysis, the in situ lidar measurement and mesoscale models can be used to estimate the atmospheric eddy dissipation rate that is relevant to aircraft wake studies.

\section{Acknowledgments}

This work was sponsored by the National Aeronautics and Space Administration's Airspace Systems Program. The work was completed under the NASA NRA "Wake Vortex Data Collection for Robust Modeling Validation to Enable Advanced, NextGen, Wake-Conscious, Capacity-Enhancing Concepts." We thank the NASA contract technical monitor, Mr. Neil O'Connor, for his support and encouragement.

\section{References}

${ }^{1}$ Campbell, S.D., et al., "Wake vortex field measurement program at Memphis, TN data guide", Lincoln Laboratory, Massachusetts Institute of Technology Project Report NASA/L-2. 1997.

${ }^{2}$ Han, J., S. P. Arya, S. Shen, and Y. Lin, "An estimation of turbulent kinetic energy and energy dissipation rate based on atmospheric boundary layer similarity theory", National Aeronautics and Space Administration Report NASA-CR-2000-210298. 2000. 
${ }^{3}$ Paquin, J. E. and S. Pond, "The determination of the Kolmogoroff constants for velocity, temperature and moisture from second and third order structure functions", Journal of Fluid Mechanics, Vol. 50, 1971, pp. 257-269.

${ }^{4}$ Taylor, G. I., "Statistical theory of turbulence", Royal Society of London Proceedings Series A, Vol. 151, 1935, pp. 421444.

${ }^{5}$ Vickers, D. and L. Mahrt, "Quality control and flux sampling problems for tower and aircraft data", Journal of Atmospheric and Oceanic Technology, Vol. 14, 1997, pp. 512-526.

${ }^{6}$ Mahrt, L. and D. Vickers, "Contrasting vertical structures of nocturnal boundary layers", Boundary Layer Meteorology, Vol. 105, 2002, pp. 351-363.

${ }^{7}$ O’Connor, E. J., A. J. Illingworth, I. M. Brooks, C. D. Westbrook, R. J. Hogan, F. Davies and B. J. Brooks, “A method for estimating the turbulent kinetic energy dissipation rate from a vertically-pointing Doppler lidar, and independent evaluation from balloon-borne in-situ measurements," Journal of Atmospheric and Oceanic Technology, Vol. 27, 2010, pp. 1652-1664.

${ }^{8}$ Frehlich, R., S. M. Hannon and S. W. Henderson, "Coherent Doppler lidar measurements of wind field statistics," Boundary Layer Meteorology, Vol. 86, 1998, pp. 233-256.

${ }^{9}$ Banakh, V., I. N. Smalikho, F. Köpp and C. Werner, "Measurements of turbulent energy dissipation rate with a CW Doppler lidar in the atmospheric boundary layer," Journal of Atmospheric and Oceanic Technology, Vol. 16, 1999, pp. 10441061 .

${ }^{10}$ Smalikho, I., F. Köpp and S. Rahm, "Measurement of atmospheric turbulence by $2-\mu$ m Doppler lidar", Journal of Atmospheric and Oceanic Technology, Vol. 22, 2005, pp. 1733-1747.

${ }^{11}$ Banakh, V., I. N. Smalikho, E. L. Pichugina and W. Brewer, "Representativeness of measurements of the dissipation rate of turbulence energy by scanning Doppler lidar," Atmospheric and Oceanic Optics, Vol. 23, 2010, pp. 48-54.

${ }^{12}$ Pruis, M. J. and D. P. Delisi, "Correlation of the temporal variability in the crosswind and the observation lifetime of vortices measured with a pulsed lidar," AIAA Paper 2011-3199.

${ }^{13}$ Coulter, R. L., "A case study of turbulence in the stable nocturnal boundary layer," Boundary Layer Meteorology, Vol. 52, 1990, pp. 75-91.

${ }^{14}$ Howell, J. F. and J. Sun, "Surface-layer fluxes in stable conditions," Boundary Layer Meteorology, Vol. 90, 1999, pp. 495520.

${ }^{15}$ Ahmad, N. N. and F. H. Proctor, "Estimation of eddy dissipation rates from mesoscale model simulations," AIAA Paper 2012-0429.

${ }^{16}$ Sarpkaya, T., "Decay of wake vortices of large aircraft," AIAA Journal, Vol. 36, 1998, pp. 1671-1679.

${ }^{17}$ Pruis, M. J. and D. P. Delisi, “Observation lifetime of an aircraft trailing vortex pair,” AIAA Paper 2012-0426.

${ }^{18}$ Proctor, F. H. and G. F. Switzer, "Numerical Simulation of Aircraft Trailing Vortices," 9th Conf. Aviation, Range and Aerospace Meteorology, September, 2000, Orlando, Florida, pp. 511-516.

${ }^{19}$ Delisi, D. P., "Laboratory Measurements of the Effect of Ambient Turbulence on Trailing Vortex Evolution," 44th AIAA Aerospace Sciences Meeting and Exhibition, Reno, NV, January, 2006, AIAA Paper 2006-1078. 